\title{
Acesso aos serviços de saúde bucal na atenção primária antes e durante o contexto da pandemia de COVID-19
}

Access to oral health services in primary care before and during the context of the pandemic of COVID-19 Acceso a servicios de salud bucal en atención primaria antes y durante el contexto de la pandemia de COVID-19 Waleska Fernanda Souto NÓBREGA 1 Gustavo Correia Basto da SILVA ${ }^{1}$ Danilo Vieira BARBOSA2

Sérgio dÁvila Lins Bezerra CAVALCANTI ${ }^{3}$

${ }^{1}$ Doutorando(a) em Odontologia, Programa de pós-graduação em Odontologia, Universidade Estadual da Paraíba - UEPB 58429-500 Campina Grande - PB, Brasil

${ }^{2}$ Graduado em Odontologia, Universidade Federal de Campina Grande - UFCG 58700-000 Patos - PB, Brasil

${ }^{3}$ Professor Doutor do Programa de pós-graduação em Odontologia da Universidade Estadual da Paraíba - UEPB 58429-500 Campina Grande - PB, Brasil

\section{Resumo}

Objetivo: O objetivo deste trabalho foi avaliar como se deu o acesso aos serviços odontológicos na atenção primária à saúde (APS) na Paraíba entre os anos de 2019 e 2020. Metodologia: Foram coletados do Sistema de Informação em Saúde para a Atenção Básica (SISAB) dados referentes às primeiras consultas odontológicas, tratamentos concluídos e vigilância em saúde bucal dos anos de 2019 e 2020. Após coletados os dados foram tabulados no Microsof Excel e em seguida analisados no SPSS versão 24.0. Resultados: Primeiras consultas odontológicas e tratamentos concluídos apresentaram uma queda vertiginosa entre os anos de 2019 e 2020 que pode ser atribuída às orientações de suspensão do atendimento odontológico eletivo durante a pandemia de Covid - 19. Conclusão: mudanças restritivas como a suspensão dos procedimentos eletivos, restringiu o acesso da população aos serviços odontológicos, podendo acarretar mudanças ainda não conhecidas e incomensuráveis na necessidade de tratamento, bem como na saúde bucal e qualidade de vida dos usuários do serviço.

Descritores: Vigilância Epidemiológica; Saúde Bucal; Infecções por Coronavirus.

\section{Abstract}

Objective: The objective of this study was to evaluate how dental services in primary health care (PHC) were accessed in Paraíba between 2019 and 2020. Methodology: They were collected from the Health Information System for Primary Care (SISAB) ) data referring to the first dental consultations, completed treatments and oral health surveillance in the years 2019 and 2020. After being collected, the data were tabulated in Microsof Excel and then unfavorable in SPSS version 24.0. Results: First dental consultations and treatments concluded a sharp drop between the years 2019 and 2020 that can be attributed to the guidelines for the suspension of elective dental care during a Covid pandemic - 19. Conclusion: restrictive changes such as the suspension of elective procedures, restricted the population's access to dental services, which may lead to changes that are not yet comparable and immeasurable in the need for treatment, as well as in the oral health and quality of life of service users.

Descriptors: Epidemiological Surveillance; Oral Health; Coronavirus Infections.

\section{Resumen}

Objetivo: El objetivo de este estudio fue evaluar cómo se accedió a los servicios odontológicos en la atención primaria de salud (APS) en Paraíba entre 2019 y 2020. Metodología: Se recolectaron a partir del Sistema de Información en Salud para Atención Primaria (SISAB)) datos referentes a la primeras consultas dentales, tratamientos completados y vigilancia de la salud bucal en los años 2019 y 2020. Luego de ser recolectados, los datos fueron tabulados en Microsof Excel y luego analizados en SPSS versión 24.0. Resultados: Las primeras consultas dentales y los tratamientos completados mostraron una fuerte caída entre los años 2019 y 2020 que se puede atribuir a las pautas para la suspensión de la atención odontológica electiva durante la pandemia de Covid - 19. Conclusión: cambios restrictivos como la suspensión de procedimientos electivos, restringió el acceso de la población a los servicios dentales, lo que puede generar cambios aún desconocidos e inconmensurables en la necesidad de tratamiento, así como en la salud bucal y la calidad de vida de los usuarios del servicio.

Descriptores: Vigilancia Epidemiológica; Salud Bucal; Infecciones por Coronavirus.

INTRODUÇÃO

Dadas as principais vias de transmissão do SARS-CoV-2 (vírus causador da Covid-19) como sendo a transmissão direta através de tosse, espirro e perdigotos e o contato com mucosa oral, nasal e ocular após tocar em superfícies contaminadas e considerando o aerossol (como o gerado pela caneta de alta rotação) também como importante via de transmissão, diversas instituições mundiais como o Centers for Disease Control and Prevention (CDC), a Agência Nacional de Vigilância Sanitária (Anvisa), o Conselho Federal de Odontologia (CFO) vem se pronunciando no que concerne a necessidade de mudanças na rotina dos atendimentos odontológicos ${ }^{1}$.

Dentre as mudanças supracitadas, evidencia-se que países como Brasil, Argentina e Chile, dentre outros, apostaram em postergar ou suspender o tratamento odontológico eletivo e manter apenas a atenção às urgências e emergências, desde que frente ao uso de equipamento de proteção individual (EPI) adequado, realizando a triagem de possíveis pacientes infectados e buscando evitar o uso da alta rotação no intuito em tentar reduzir a possibilidade de contaminação pelo novo coronavírus no interior do ambiente do consultório ${ }^{2}$.

Considerando-se que ainda vivemos em contexto de pandemia após mais de um ano da propositura de tais mudanças, não se sabe ao certo como essas medidas de suspenção de atendimento eletivo estão influenciando ou irão influenciar na saúde bucal da população no futuro. Logo, o objetivo desta pesquisa foi avaliar como se deu o acesso aos serviços odontológicos na atenção primária à saúde 
(APS) na Paraíba entre os anos de 2019 e 2020.

MATERIAL E MÉTODO

Foram coletados do Sistema de Informação em Saúde para a Atenção Básica (SISAB) dados referentes às primeiras consultas odontológicas, tratamentos concluídos e vigilância em saúde bucal (dor de dente e abcesso dentoalveolar) dos anos de 2019 (imediatamente anterior à pandemia) e 2020 (ano 1 da pandemia de Covid - 19) afim de que pudesse estabelecer um comparativo que demonstrasse se houve alteração significativa no acesso aos serviços odontológicos no período.

Após coletados os dados foram tabulados no Microsof Excel e em seguida analisados no SPSS versão 24.0. Foi realizado o cálculo do percentual de variação $(\% \mathrm{~V})$ entre os anos coletados, em seguida, considerandose a normalidade dos dados, foi realizado o Teste $T$ de Student para se verificar diferenças estatisticamente relevantes entre os dados.

RESULTADOS E DISCUSSÃO

Na Tabela 1 pode-se perceber que, entre o ano de 2019 e o ano de 2020, as primeiras consultas odontológicas e os tratamentos odontológicos concluídos apresentaram uma queda vertiginosa no estado da Paraíba, identificadas em $42,3 \%$ e $46,8 \%$ respectivamente. Os anos avaliados apresentaram diferença estatisticamente relevante entre si, logo, esta não pode ser atribuída ao acaso.

Tabela 1. Análise da diferença entre as primeiras consultas odontológicas e tratamentos concluídos realizados nos anos de 2019 e 2020, Paraíba, Brasil - 2021.

\begin{tabular}{|c|c|c|c|c|c|c|c|c|}
\hline \multirow{2}{*}{$\begin{array}{l}\text { Variável } \\
\text { Mês/Ano }\end{array}$} & \multicolumn{4}{|c|}{$\begin{array}{c}\text { Primeiras consultas } \\
\text { odontológicas }\end{array}$} & \multicolumn{4}{|c|}{ Tratamentos concluídos } \\
\hline & 2019 & 2020 & $\% V^{*}$ & $p^{* * *}$ & 2019 & 2020 & $\% V^{*}$ & $p^{* * *}$ \\
\hline Janeiro & 32407 & 32089 & & & 12012 & 12059 & & \\
\hline Fevereiro & 36867 & 30961 & & & 14056 & 12854 & & \\
\hline Março & 27980 & 24558 & & & 10933 & 9881 & & \\
\hline Abril & 30856 & 3500 & & & 13192 & 973 & & \\
\hline Maio & 36361 & 4360 & & & 15211 & 1330 & & \\
\hline Junho & 25148 & 5648 & 42,3 & 0,001 & 10683 & 1887 & 46,8 & $<0,001$ \\
\hline Julho & 35257 & 12177 & & & 14688 & 3760 & & \\
\hline Agosto & 34752 & 17658 & & & 14605 & 6143 & & \\
\hline Setembro & 35760 & 24927 & & & 16104 & 8534 & & \\
\hline Outubro & 35877 & 26809 & & & 15985 & 12260 & & \\
\hline Novembro & 31142 & 22982 & & & \begin{tabular}{|l|}
14767 \\
\end{tabular} & 9894 & & \\
\hline Dezembro & 19562 & 15158 & & & \begin{tabular}{|l|}
10971 \\
\end{tabular} & 7158 & & \\
\hline
\end{tabular}

A redução considerável do número de primeiras consultas e tratamentos concluídos pode-se ser explicada pelas orientações dadas em conjunto pela Associação de Medicina Intensiva Brasileira (AMIB) e Conselho Federal de Odontologia (CFO) publicadas em fevereiro de 2020 orientando que, mesmo frente aos pacientes sem suspeita para Covid - 19, se suspendesse o tratamento odontológico eletivo ${ }^{3}$.

Salienta-se que, ao utilizar-se o indicador da razão entre primeiras consultas e tratamentos concluídos proposto pelo Ministério da Saúde ${ }^{4}$, ambos os anos apresentam um número satisfatório (>50\%). Logo, aconselha-se que para avaliar os impactos pós-pandemia e planejar melhorias para os serviços e saúde bucal da população, este número não seja avaliado isoladamente, mas em série histórica.

Ao observar-se o campo da vigilância epidemiológica em saúde bucal, no que concerne à dor de dente e a abcessos dentoalveolares, consideradas urgências odontológicas, verificou-se queda pouco expressiva nos números, respectivamente $6 \mathrm{e}$ $10 \%$, consideradas não relevantes estatisticamente $(p>0,05)$.

Tabela 2. Análise da vigilância epidemiológica em saúde bucal para os anos de 2019-2020, Paraíba, Brasil - 2021.

\begin{tabular}{|c|c|c|c|c|c|c|c|c|}
\hline \multirow{2}{*}{$\begin{array}{l}\text { Variável } \\
\text { Mês/Ano } \\
\end{array}$} & \multicolumn{4}{|c|}{ Dentalgia } & \multicolumn{4}{|c|}{ Abscesso dentário } \\
\hline & 2019 & 2020 & \% $\mathbf{V}^{*}$ & $\mathbf{P}^{* * *}$ & 2019 & 2020 & $\% V^{*}$ & $p^{* *}$ \\
\hline Janeiro & 11032 & 10211 & & & 803 & 732 & & \\
\hline Fevereiro & 11986 & 9778 & & & 930 & 779 & & \\
\hline Março & 10067 & 9579 & & & 734 & 729 & & \\
\hline Abril & 12116 & 6015 & & & 881 & 448 & & \\
\hline Maio & 13024 & 7689 & & & 1005 & 513 & & \\
\hline Junho & 9548 & 8838 & 6,3 & 0,490 & 667 & 559 & 10,6 & 0,335 \\
\hline Julho & 12828 & 14215 & & & 887 & 811 & & \\
\hline Agosto & 13486 & 14176 & & & 922 & 857 & & \\
\hline Setembro & 12953 & 15488 & & & 945 & 1024 & & \\
\hline Outubro & 14050 & 14741 & & & 1005 & 1087 & & \\
\hline Novembro & 12041 & 12504 & & & 867 & 898 & & \\
\hline Dezembro & 7622 & 8687 & & & 574 & 699 & & \\
\hline
\end{tabular}

Vale salientar que as recomendações AMIB/CFO mantiveram 0 atendimento a urgências e emergências durante o contexto de pandemia, desde que se atentasse às preocupações corriqueiras e adicionais para a equipe de saúde bucal quanto ao uso de EPI adequado, limpeza e desinfecção do equipo e periféricos e descarte do material contaminado, principalmente ${ }^{3}$.

Os dados aqui apresentados contrastam com o preprint publicado por Lucena et al. ${ }^{5}$, que, ao avaliar o primeiro quadrimestre de atendimentos de 2019-2020 no país, também encontraram queda no número de consultas marcadas por dentalgia (dor de dente) e abcesso dentoalveolar, sugerindo a investigação que demonstre se as urgências realmente diminuíram ou se estas não foram acolhidas pelo serviço público.

CONCLUSÃO

Frente a uma nova doença infecciosa, inesperada, e que rapidamente atingiu 0 contexto de pandemia, as autoridades médicas e odontológicas precisaram, com urgência, adotar medidas orientadoras para o atendimento odontológico, buscando que este não contribuísse para o aumento da infecção e número de casos da doença. No entanto, mudanças restritivas como a suspensão dos procedimentos eletivos, restringiu o acesso da população aos serviços odontológicos, podendo 
acarretar mudanças ainda não conhecidas e incomensuráveis na necessidade de tratamento, bem como na saúde bucal e qualidade de vida dos usuários do serviço.

\section{REFERÊNCIAS}

1. Baldan, LC, Teixeira FF, Zermiani, T.C. Atenção odontológica durante a pandemia de COVID-19: uma revisão de literatura. Vigil sanit debate. 2021;9(1):36-46.

2. Carrer FCA, Galante ML, Gabriel M, Pischel N, Giraldes AI, Neumann A et al. A COVID-19 na América Latina e suas repercussões para a odontologia. Rev Panam Salud Publica. 2020;44:20202020.

3. Associação de Medicina Intensiva Brasileira (AMIB). Conselho Federal de Odontologia (CFO). Recomendações AMIB/CFO para enfrentamento da COVID-19 na Odontologia. Fevereiro. [Internet]. 2020. [citado em 03 de Maio de 2021]. Disponível em: https://website.cfo.org.br/wpcontent/uploads/2020/03/AMIB_CFORecomendac\%CC\%A70\%CC\%83es.pdf.

4. Santiago CP, Cavalcante DFB, Ambrosano GMB, Pereira AC, Lucena EHG, Cavalcanti YW et al. Resolutividade da Atenção Básica em Saúde Bucal em municípios do estado da Paraíba, Brasil. Ciênc Saude Coletiva. 2021;26(Supl 2):3589-97.

5. Lucena EHG, Freire AR, Freire DEWG, Araújo ECF, Lira GNW, Brito ACM et al. Acesso em saúde bucal na atenção básica antes e após 0 início da pandemia de COVID19 no Brasil. PBOCI - 2020 -0139. (preprint). [Citado em 03 de Maio de 2021]. Disponível em: https://preprints.scielo.org/index.php/scielo/prep rint/view/819/1126.

\section{CONFLITO DE INTERESSES}

Os autores declaram não haver conflitos de interesse

AUTOR PARA CORRESPONDÊNCIA

\section{Waleska Fernanda Souto Nóbrega}

Rua Baraúnas, 351 - Universitário,

58429-500 Campina Grande - PB, Brasil

E-mail:waleska_bic@hotmail.com

Submetido em 23/05/2021

Aceito em 16/07/2021 